\title{
Gankyrin promotes epithelial-mesenchymal transition and metastasis in NSCLC through forming a closed circle with IL-6/ STAT3 and TGF- $\beta /$ SMAD3 signaling pathway
}

\author{
Wu-ping Wang ${ }^{1, *}$, Ying Sun ${ }^{1, *}$, Qiang Lu, ${ }^{1, *}$ Jin-bo Zhao ${ }^{1}$, Xue-jiao Wang ${ }^{1}$, Zhao \\ Chen ${ }^{1}$, Yun-feng $\mathrm{Ni}^{1}$, Ju-zheng Wang ${ }^{1}$, Yong Han ${ }^{1}$, Zhi-pei Zhang ${ }^{1}$, Xiao-long Yan ${ }^{1}$, \\ Xiao-fei Li ${ }^{1}$ \\ ${ }^{1}$ Department of Thoracic Surgery, Tangdu Hospital, The Fourth Military Medical University, Xi'an, 710038, China \\ *These authors contributed equally to this work
}

Correspondence to: Zhi-pei Zhang, email: zzpzyy@fmmu.edu.cn

Xiao-long Yan, email: yanxiaolong@fmmu.edu.cn

Xiao-fei Li, email: Ixfchest@fmmu.edu.cn

Keywords: NSCLC, gankyrin, EMT, metastasis, closed circle

Received: September 02, 2016

Accepted: December 08, 2016

Published: December 15, 2016

\section{ABSTRACT}

Our previous research showed that Gankyrin was overexpressed in NSCLC and significantly associated with clinicopathologic features and poor prognosis. In this study, we will explore potential effect of Gankyrin on EMT and metastasis in NSCLC. The ectopic higher expression of Gankyrin markedly increased the migration and invasion in NSCLC cells. In contrast, silencing Gankyrin inhibit this aggressive behavior in NSCLC cells. Further study demonstrated that overexpression of Gankyrin could decrease E-cadherin expression and increase expression of Vimentin and Twist1 at mRNA and protein levels. These data indicated that Gankyrin could facilitate occurrence and development of EMT. Also IHC analysis showed that Gankyrin expression was negatively correlated with E-cadherin expression, while positively correlated with Vimentin and Twist1 expression in NSCLC tissues. The mechanism study finally suggested that the Gankyrin-driven EMT was partially due to IL-6/pSTAT3 and TGF- $\beta /$ p-SMAD3 pathways activation. Taken together, our data provided a novel mechanism of Gankyrin promoting EMT and metastasis in NSCLC through forming a closed circle with IL-6/p-STAT3 and TGF- $\beta$ /p-SMAD3 signaling pathway.

\section{INTRODUCTION}

Lung cancer is responsible for approximately 1.4 million deaths annually $[1,2]$. According to the World Health Organization (WHO)'s estimates, more than one million Chinese will be diagnosed with lung cancer every year by 2025 , thus the death toll will increase accordingly [3]. In lung cancer, approximately $85 \%$ are non-small cell lung cancer (NSCLC) [4], and a majority of NSCLC patients die from local recurrence or distant metastasis even after undergoing curative surgical resection, thus the 5-year survival rate is still unsatisfactory [5]. Therefore, there is an urgent requirement to understand the metastatic mechanism in NSCLC for targeted therapy and prognosis.

Since the report of the pivotal role of the epithelialmesenchymal transition (EMT) during embryonic development in the 1980s, many studies have demonstrated that EMT plays essential roles in tumor progression, especially in tumor metastasis $[6,7]$. In lung cancer, the EMT has been recognized as an initial step and critical procedure of tumor cell metastasis [8-10]. Signaling pathways, such as IL-6/STAT3, TGF- $3 /$ SMAD3, PI3K/ $\mathrm{AKT}$ and et al, can induce EMT during development and differentiation [11, 12]. However, the mechanisms underlying EMT induction in cancer cells remain unclear.

Gankyrin (also known as PSMD 10 or p28GANK) was initially purified and characterized by Tanaka and coworkers in 1998 [13]. Recently, it was identified as an oncoprotein frequently overexpressed in several cancers [14-19]. We also tested Gankyrin expression in NSCLC in preliminary study [20]. The results showed that the gene and protein of Gankyrin expressed higher in human 
NSCLC tissues than in the corresponding adjacent noncancerous tissues, and Gankyrin overexpression was significantly associated with lymph node metastasis and poor prognosis. These data indicated that Gankyrin might be a conservative molecule playing essential roles in tumor progression. However, until now, the exact mechanisms of Gankyrin in NSCLC progression still remain unknown. Our previous study also showed an apparent prognostic significance of Gankyrin overexpression in early stage patients, but no prognostic significance in late stage patients [20], which suggested that Gankyrin might participate in the initial step of NSCLC metastasis, such as the EMT process.

Both the IL- 6 and TGF- $\beta$ are important cytokines initiating EMT in tumor microenvironment. However, once the tumor cells acquire the EMT phenotype induced by IL- 6 and TGF- $\beta$, they leave away from the primary sites of the microenvironment. The concentrations of those cytokines surrounding the cells reduce rapidly, so what are the mechanisms for the cells to sustain the EMT phenotypes till they migrate and locate in a new metastatic site. We guess that there are some key proteins to connect the downstream signal molecules of IL- 6 and TGF- $\beta$, such as phosphorylated STAT3 (p-STAT3) and phosphorylated SMAD3 (p-SAMD3), forming a closed circle to sustain the EMT phenotype $[21,22]$. Our preliminary experimental results show that Gankyrin expression was correlated with IL-6 and TGF- $\beta$, so we speculate that Gankyrin may be one of them.

In the present study, we demonstrated that Gankyrin was upregulated in NSCLC tissues and cell lines. Overexpressed Gankyrin increases NSCLC migration and invasion, and silencing Gankyrin inhibited NSCLC migration and invasion. Further study demonstrated that over-expression of Gankyrin could decrease expression of E-cadherin and increase expression of Vimentin and Twist1, and silencing Gankyrin expression could increase expression of E-cadherin and decrease expression of

A

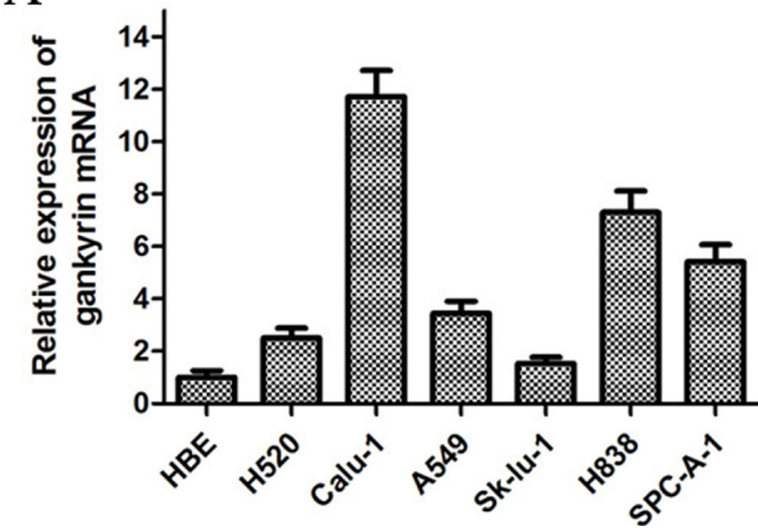

Vimentin and Twist1. Furthermore, our study provided a new mechanism that Gankyrin-promoted EMT and metastasis in NSCLC was partially due to forming a closed circle with IL-6/p-STAT3 and TGF- $\beta / \mathrm{p}-\mathrm{SMAD} 3$ signaling pathways.

\section{RESULTS}

\section{Gankyrin was over-expressed in human NSCLC cell lines}

We assessed Gankyrin expression in six human NSCLC cell lines (A549, Sk-lu-1, SPC-A-1, H838, H520, Calu-1) and one normal human bronchial epithelium cell line (HBE) using Semi-quantitative RT-PCR and Western blot assay. As shown in Figure $1 \mathrm{~A}$ and 1B, Gankyrin expression was higher in the NSCLC cell lines (H520, Calu-1, A549, Sk-lu-1, H838, SPC-A-1) when compared with its expression in the $\mathrm{HBE}$ cell line both at the mRNA and protein levels.

\section{Silencing Gankyrin inhibited NSCLC cell migration and invasion}

Our previous results revealed that high level of Gankyrin was associated with poor prognosis in NSCLC patients, but the underlying mechanisms remain unclear. Thus, to find out whether Gankyrin was associated with poor prognosis of NSCLC patients through promoting tumor metastasis, we applied lenti-virus transduction to knockdown the Gankyrin expression in Calu-1 and H838 cell lines (Figure 2A and 2B) and then detected the migratory and invasive ability of these cell lines, in which the Gankyrin expression was relatively higher than that in other NSCLC cell lines. The results suggested that the disruption of Gankyrin expression could significantly inhibit the migratory and invasive ability of Calu-1 and H838 cells by wounding healing (Figure 2C and 2D) and

B

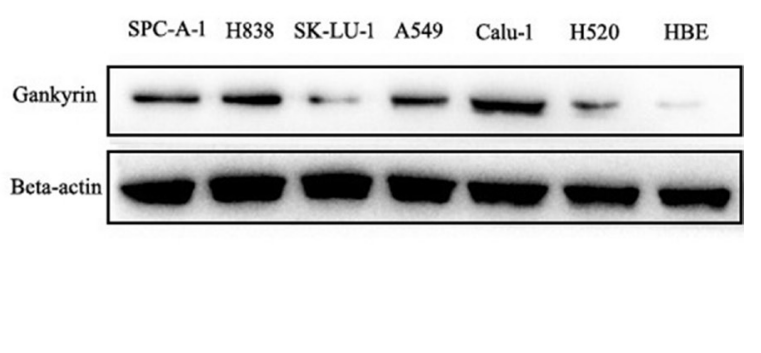

Figure 1: Gankyrin mRNA and protein expressions in six human NSCLC and normal bronchial epithelium cell lines. (A) Gankyrin mRNA expression was higher in human NSCLC cell lines than HBE cell line. (B) Gankyrin protein expression was higher in human NSCLC cell lines than HBE cell line. 
transwell migration (Figure 2E and 2F) and invasion assay (Figure 2G and 2H).

\section{Gankyrin overexpression could promote the NSCLC cell migration and invasion}

To further prove the role of Gankyrin in NSCLC metastasis, we stably overexpressed Gankyrin expression in H520 and Sk-lu-1 cell lines, in which the Gankyrin expression is relatively lower than that in the other NSCLC cell lines (Figure 3A and 3B). Wounding healing and transwell assay showed that Gankyrin overexpression could significantly increase the migratory and invasive ability of H520 and Sk-lu-1 cells (Figure 3C-3H). These findings support the hypothesis that Gankyrin plays an important role in promoting NSCLC metastasis.

A

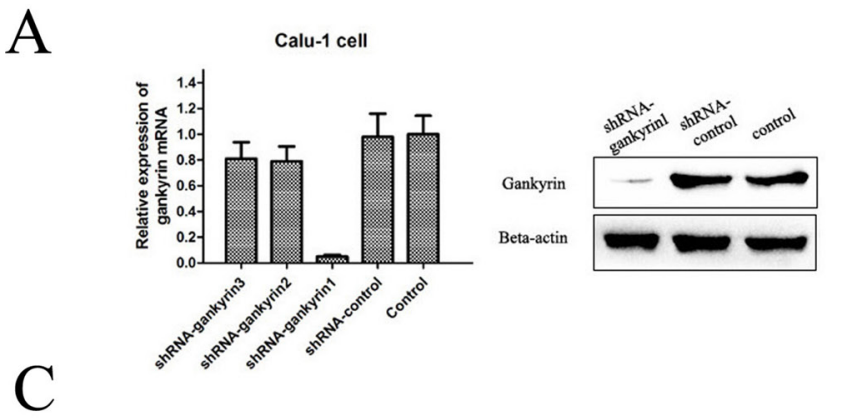

$\mathrm{C}$

B

\section{Gankyrin could induce the EMT phenotypes in NSCLC cell lines}

To further explore potential mechanisms of Gankyrin associated with metastasis in NSCLC, essential EMTrelated biomarkers including E-cadherin, Vimentin and Twist1 were tested by quantitative RT-PCR and Western blot assay. We found that lower mRNA and protein levels of mesenchymal markers were expressed in Gankyrin knockdown cells lines (Calu-1-shRNA-Gankyrin and H838-shRNA-Gankyrin), such as Vimentin and Twist1, whereas higher mRNA and protein levels of the epithelial marker of E-cadherin were observed (Figure 4A-4D). In addition, we observed increased expression of the epithelial marker E-cadherin and decreased expression of mesenchymal markers Vimentin, Twist in Gankyrin

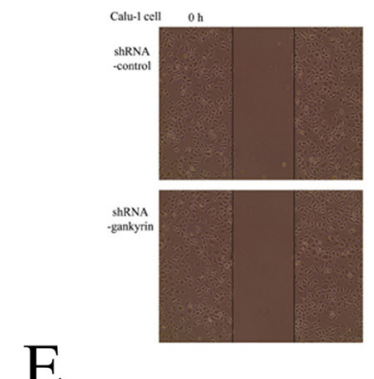

E

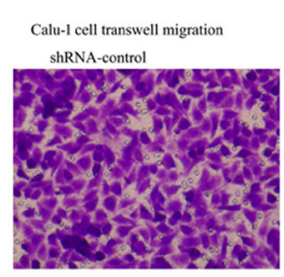

G

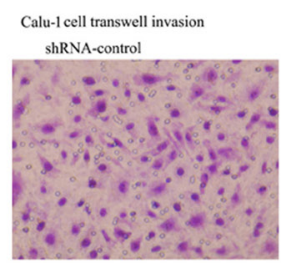

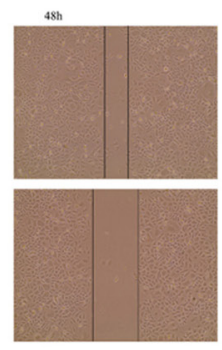
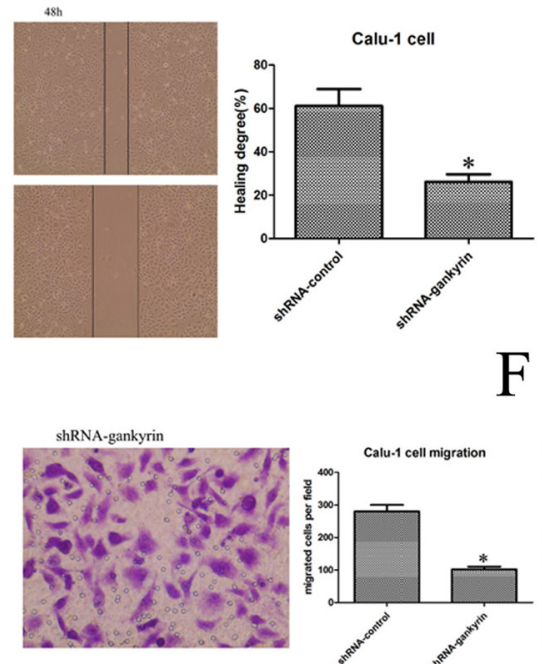

$\mathrm{F}$
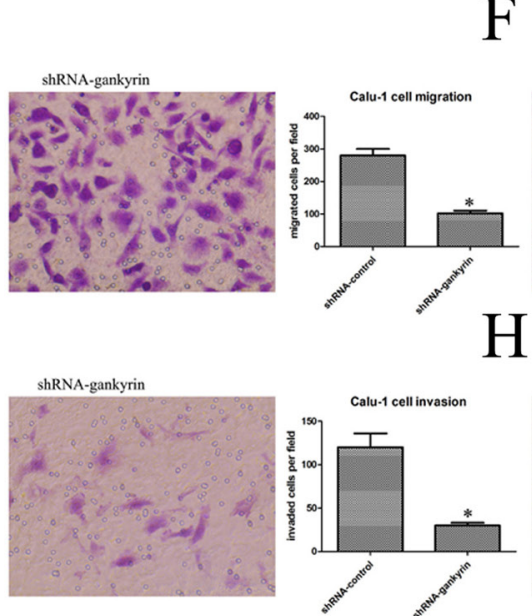

$\mathrm{H}$
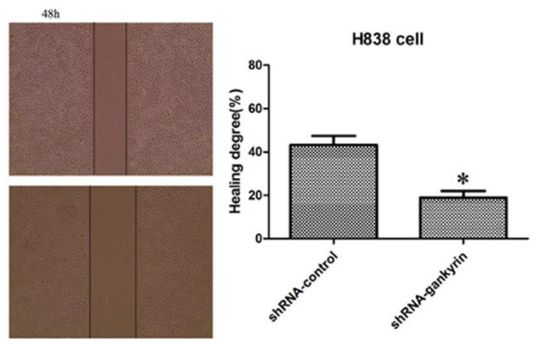

H838 cell transwell migration
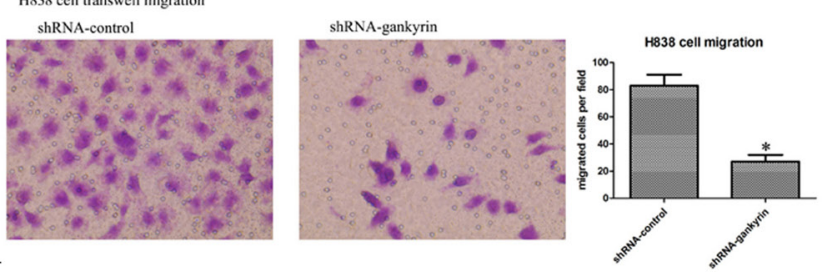

H838 cell transwell invasion
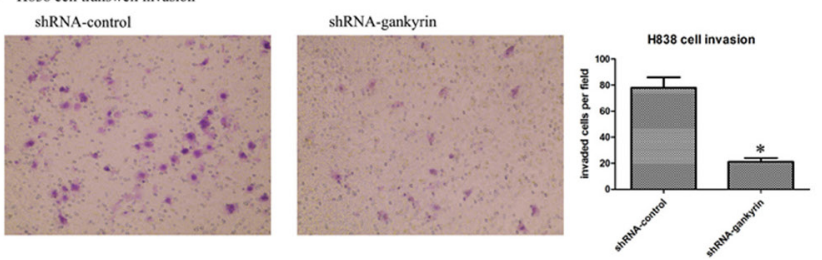

Figure 2: Silencing Gankyrin inhibited NSCLC cell migration and invasion. (A) and (B) Lenti-virus transduction decreased the Gankyrin expression in Calu-1 and H838 cell lines. (C) and (D) Scratch wound healing assay showed that knockdown of Gankyrin inhibited the migration of Calu-1 and H838 cell lines. (E) and (F) Transwell migration assay showed that knockdown of Gankyrin inhibited the migration of Calu-1 and H838 cell lines. $(\mathbf{G})$ and $(\mathbf{H})$ Transwell assay showed that knockdown of Gankyrin inhibited the invasion of Calu- 1 and H838 cell lines. $* P<0.05$. 
knockdown cells lines by immunoblotting assay (Figure 5A). In line with the above observations, when Gankyrin was stably overexpressed in H520 and Sk-lu-1 cells, we found that both H520 pCDNA3.1-Gankyrin cells and Sk-lu-1 pCDNA3.1-Gankyrin cells expressed higher mRNA and protein levels of mesenchymal markers, such as Vimentin and Twist1, whereas lower mRNA and protein levels of the epithelial marker of E-cadherin were observed (Figure 4E-4H). Immunoblotting assay in Gankyrin overexpressed cell lines also showed increased expression of the mesenchymal markers Vimentin, Twist 1 and decreased expression of the epithelial marker E-cadherin (Figure 5B). Together, these observations showed that Gankyrin might induce EMT and metastasis in NSCLC cells.

\section{IHC results showed that Gankyrin expression correlated with EMT-related biomarkers in NSCLC tissues}

We used immunohistochemistry (IHC) to analyze the frequency of three EMT-related biomarkers (E-cadherin, Vimentin and Twist1) in the same investigation cohort as Gankyrin expression was studied. Representative microphotographs of the expression of these three biomarkers in ADC and SCC tissues are shown in Figure $6 \mathrm{~A}$ and $6 \mathrm{~B}$. The percentage of positive expression of E-cadherin, Vimentin and Twist1 in Gankyrin-positive tumors and Gankyrin-negative tumors in ADC and SCC tissues were shown in Tables 1 and 2, respectively. Correlation analysis showed that Gankyrin expression in
A

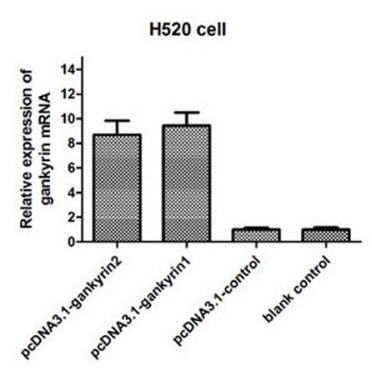

$\mathrm{C}$
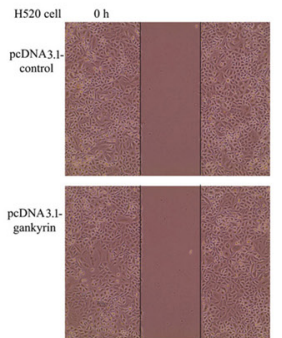

$\mathrm{E}$

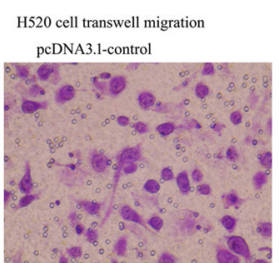

$\mathrm{G}$

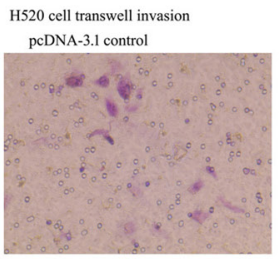

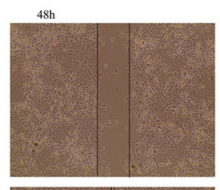
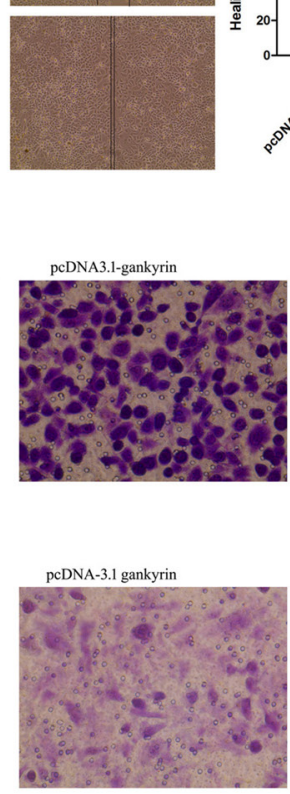
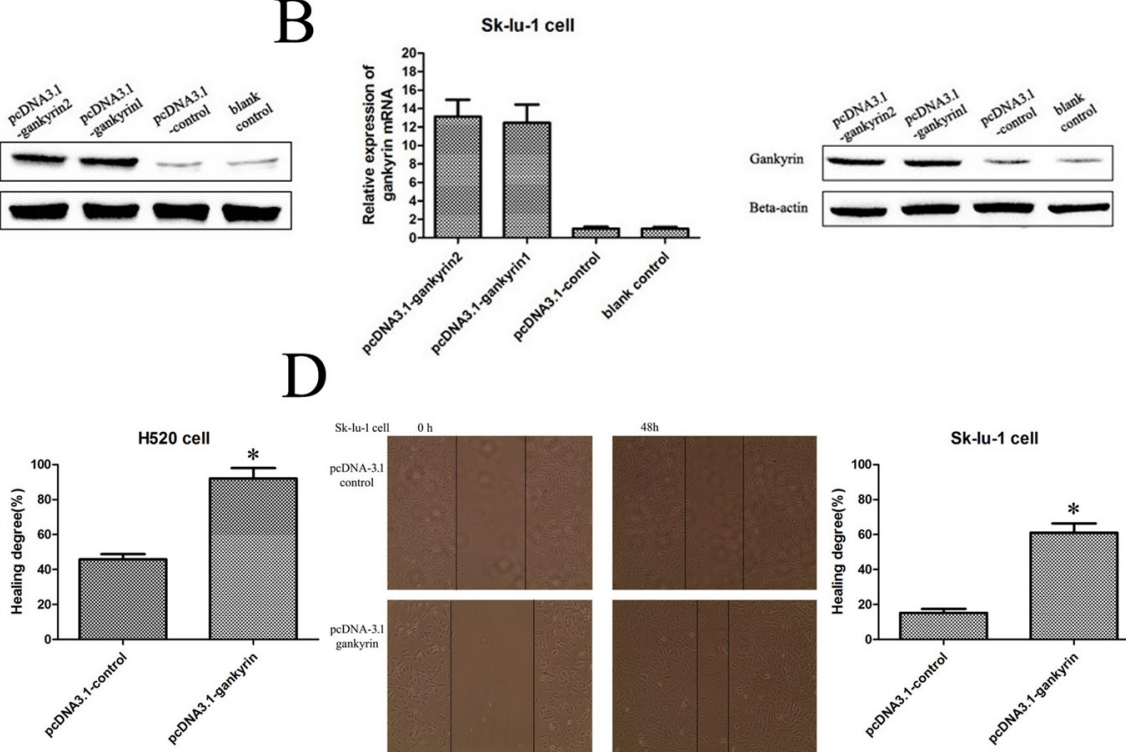

$\mathrm{D}$
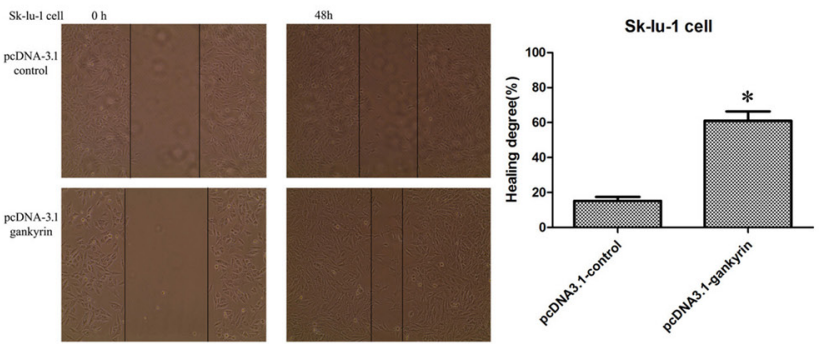

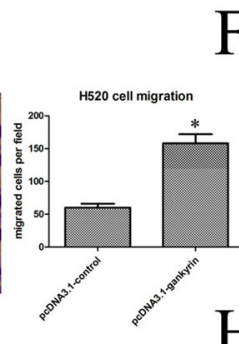

Sk-lu-l cell transwell migration pcDNA-3.1 contro
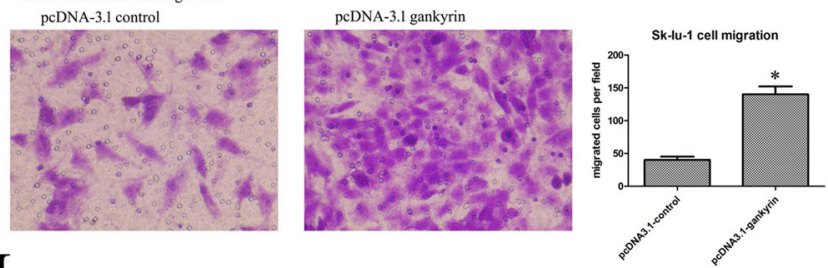

$\mathrm{H}$

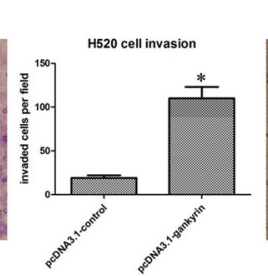

Sk-lu-1 cell transwell invasion
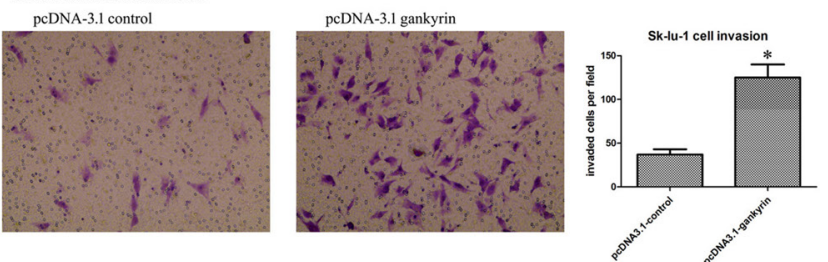

Figure 3: Gankyrin over-expression could promote the NSCLC cell migration and invasion. (A) and (B) Plasmid transduction promoted the Gankyrin expression in H520 and SK-LU-1 cell lines. (C) and (D) Scratch wound healing assay showed that overexpession of Gankyrin promoted the migration of H520 and SK-LU-1 cell lines. (E) and (F) Transwell migration assay showed that overexpession of Gankyrin promoted the migration of H520 and SK-LU-1 cell lines. (G) and (H) Transwell assay showed that overexpession of Gankyrin promoted the invasion of H520 and SK-LU-1 cell lines. ${ }^{*} P<0.05$. 
Table 1: Correlation of Gankyrin expression with E-cadherin, vimentin and twist1 in ADC tissues

\begin{tabular}{|c|c|c|c|c|}
\hline \multirow[t]{2}{*}{ ADC tissues } & \multicolumn{2}{|c|}{ Gankyrin expression } & \multirow[t]{2}{*}{ Correlation coefficient } & \multirow[t]{2}{*}{$P$-value } \\
\hline & Positive (\%) & Negative (\%) & & \\
\hline E-cadherin expression & & & $r=-0.340$ & 0.009 \\
\hline Positive (\%) & 23 & 8 & & \\
\hline Negative (\%) & 36 & 1 & & \\
\hline Vimentin expression & & & $r=0.290$ & 0.027 \\
\hline Positive (\%) & 38 & 2 & & \\
\hline Negative $(\%)$ & 21 & 7 & & \\
\hline Twist1 expression & & & $r=0.347$ & 0.007 \\
\hline Positive (\%) & 42 & 3 & & \\
\hline Negative (\%) & 17 & 6 & & \\
\hline
\end{tabular}

Table 2: Correlation of Gankyrin expression with E-cadherin, vimentin and twist1 in SCC tissues

\begin{tabular}{|c|c|c|c|c|}
\hline \multirow[t]{2}{*}{ SCC tissues } & \multicolumn{2}{|c|}{ Gankyrin expression } & \multirow[t]{2}{*}{ Correlation coefficient } & \multirow[t]{2}{*}{$P$-value } \\
\hline & Positive (\%) & Negative (\%) & & \\
\hline E-cadherin expression & & & $r=-0.250$ & 0.018 \\
\hline Positive (\%) & 34 & 18 & & \\
\hline Negative (\%) & 40 & 6 & & \\
\hline Vimentin expression & & & $r=0.251$ & 0.017 \\
\hline Positive (\%) & 49 & 9 & & \\
\hline Negative (\%) & 25 & 15 & & \\
\hline Twist1 expression & & & $r=0.247$ & 0.024 \\
\hline Positive (\%) & 54 & 11 & & \\
\hline Negative (\%) & 20 & 13 & & \\
\hline
\end{tabular}

ADC or SCC tissues had positive correlations with the expression of Vimentin $(r=0.290, P=0.027 ; r=0.251$ $P=0.017)$ and Twist1 $(r=0.347, P=0.007 ; r=0.247$ $P=0.024)$, while negative correlations with the E-cadherin expression $(r=-0.340, P=0.009 ; r=-0.250$, $P=0.018$ ). These data strongly supported that Gankyrin was associated with EMT in NSCLC.

\section{Gankyrin-promoted EMT is partially due to IL-6/p-STAT3 and TGF-ק/p-SMAD3 pathways activation}

To explore whether Gankyrin could be regulated by TGF- $\beta 1$ in NSCLC, Sk-lu-1 cells were exposed to TGF- $\beta 1$ stimulation for 3 days (concentration from $0-10 \mathrm{ng} / \mathrm{ml}$ ). p-SMAD3 and Gankyrin were evaluated by Western blot assay. The results showed that the expressions of p-SMAD3 and Gankyrin were higher increased along with TGF- $\beta 1$ concentration, which indicated that Gankyrin could be regulated by TGF- $\beta 1$ (Figure 7A). To further explore whether Gankyrin could be regulated by
TGF- $\beta 1$ through p-SMAD3 in NSCLC, we applied siRNA transduction to knockdown the SMAD3 expression in Sklu- 1 cell lines, then the cells were exposed to TGF- $\beta 1$ stimulation for 3 days (concentration from $0-10 \mathrm{ng} / \mathrm{ml}$ ), and the p-SMAD3 and Gankyrin were evaluated by Western blot assay. The results showed that there were not any changes on p-SMAD3 and Gankyrin expressions when siRNA-SMAD3 Sk-lu-1 cell lines were exposed to TGF- $\beta 1$ stimulation, which indicated that Gankyrin could be regulated by TGF- $\beta 1$ through p-SMAD3 (Figure 7B). As we have shown that Gankyrin could be regulated by TGF- $\beta 1$ in NSCLC, and TGF- $\beta 1$ absolutely could induce EMT, we investigated whether Gankyrin also participated in the TGF- $\beta 1$-induced EMT process. Interestingly, the data showed that the Gankyrin overexpression did markedly induce EMT with TGF- $\beta 1$; On the other hand, when Gankyrin expression in Sk-lu-1 pCDNA3.1Gankyrin cells was knockdown, the TGF- $\beta 1$-induced EMT phenotype reduced rapidly, which further supported that Gankyrin participated in the TGF- $\beta 1$-induced EMT process (Figure $7 \mathrm{C}$ ). In order to strongly confirm this 
A

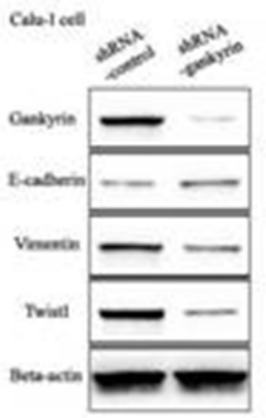

$\mathrm{C}$

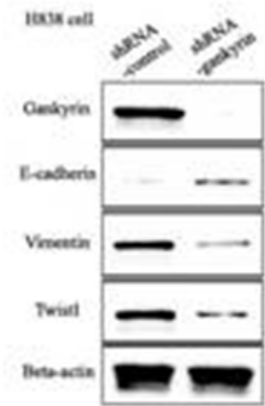

E

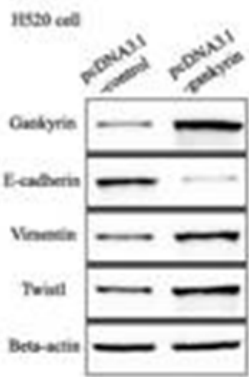

G

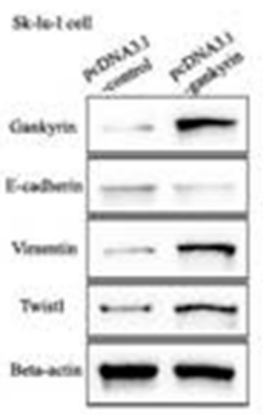

B

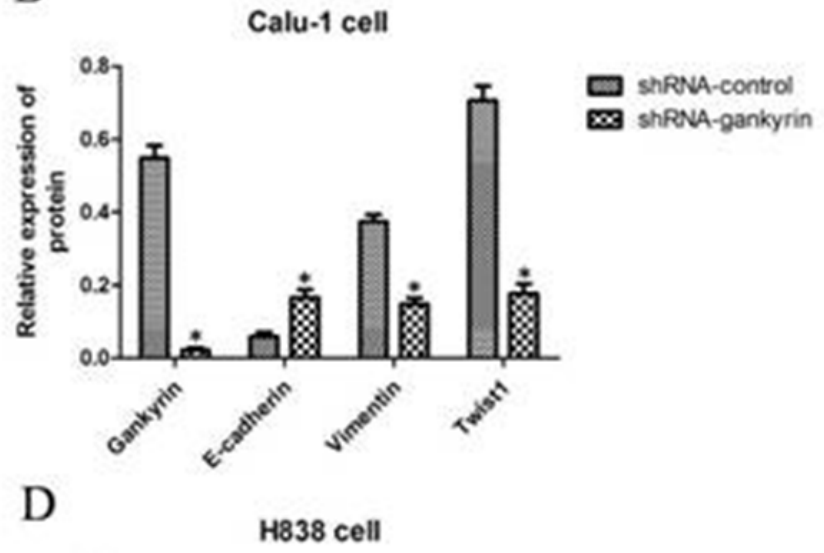

H838 cell

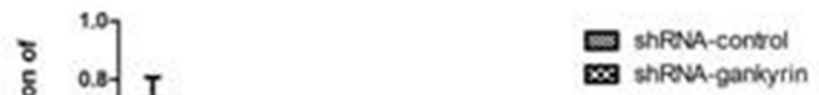

$\mathrm{F}$

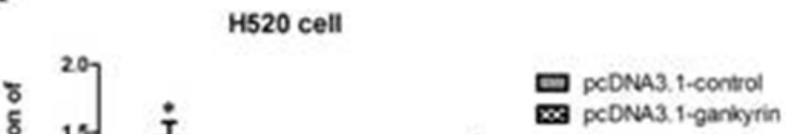

$\mathrm{H}$

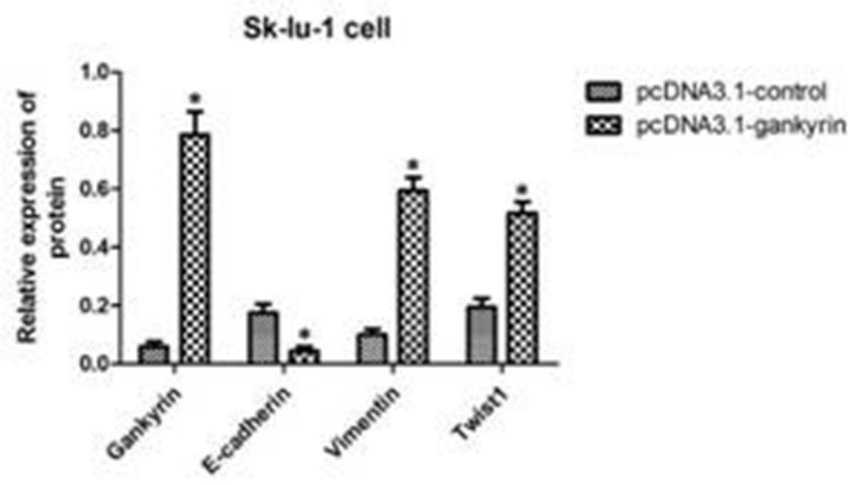

Figure 4: EMT-related biomarkers including E-cadherin, Vimentin and Twist1 were tested by qRT-PCR and western blot assays. (A) and (B) E-cadherin, Vimentin and Twist1 mRNA and protein expressions in Gankyrin decreased Calu-1 cell line. (C) and (D) E-cadherin, Vimentin and Twist1 mRNA and protein expressions in Gankyrin decreased H838 cell line. (E) and (F) E-cadherin, Vimentin and Twist1 mRNA and protein expressions in Gankyrin increased H520 cell line. (G) and (H) E-cadherin, Vimentin and Twist1 mRNA and protein expressions in Gankyrin increased SK-LU-1 cell line. ${ }^{*} P<0.05$. 
Table 3: Correlation of Gankyrin expression with p-SMAD3 and p-STAT3 in all NSCLC tissues

\begin{tabular}{|c|c|c|c|c|}
\hline \multirow[t]{2}{*}{ NSCLC tissues } & \multicolumn{2}{|c|}{ Gankyrin expression } & \multirow[t]{2}{*}{ Correlation coefficient } & \multirow[t]{2}{*}{$P$-value } \\
\hline & Positive (\%) & Negative (\%) & & \\
\hline P-SMAD3 & & & $r=0.346$ & 0.001 \\
\hline Positive (\%) & 42 & 5 & & \\
\hline Negative $(\%)$ & 30 & 21 & & \\
\hline P-STAT3 & & & $r=0.322$ & 0.002 \\
\hline Positive (\%) & 37 & 4 & & \\
\hline Negative (\%) & 35 & 22 & & \\
\hline
\end{tabular}

A
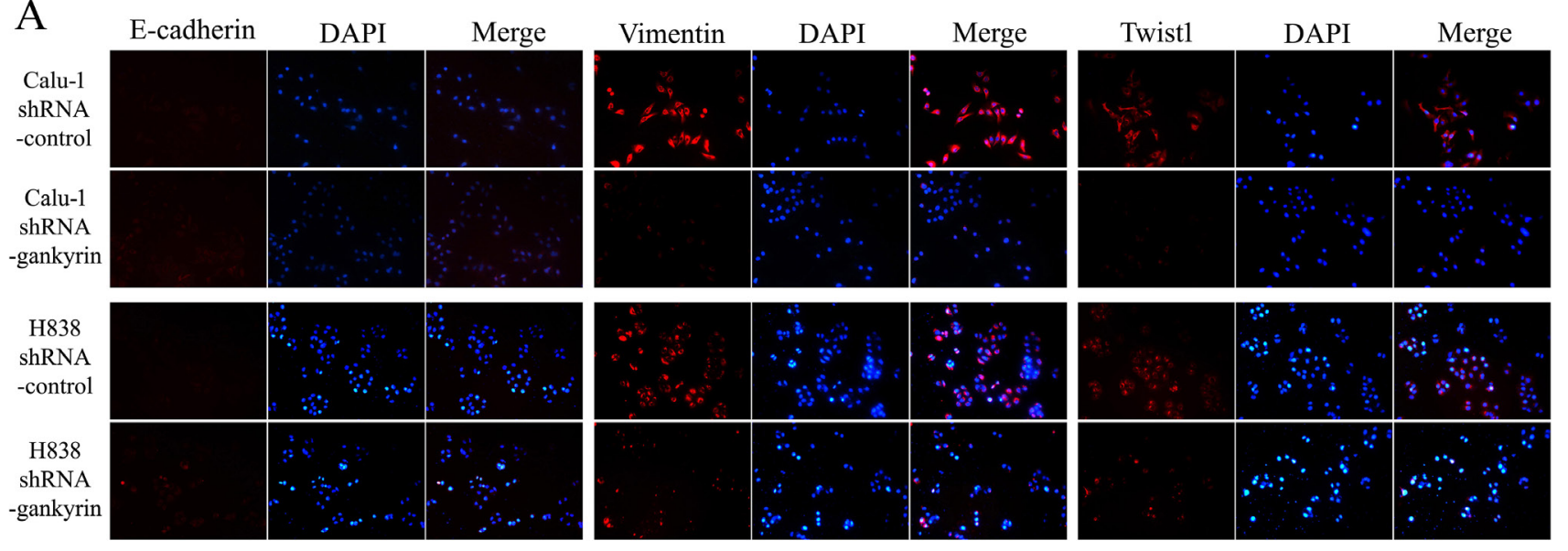

B
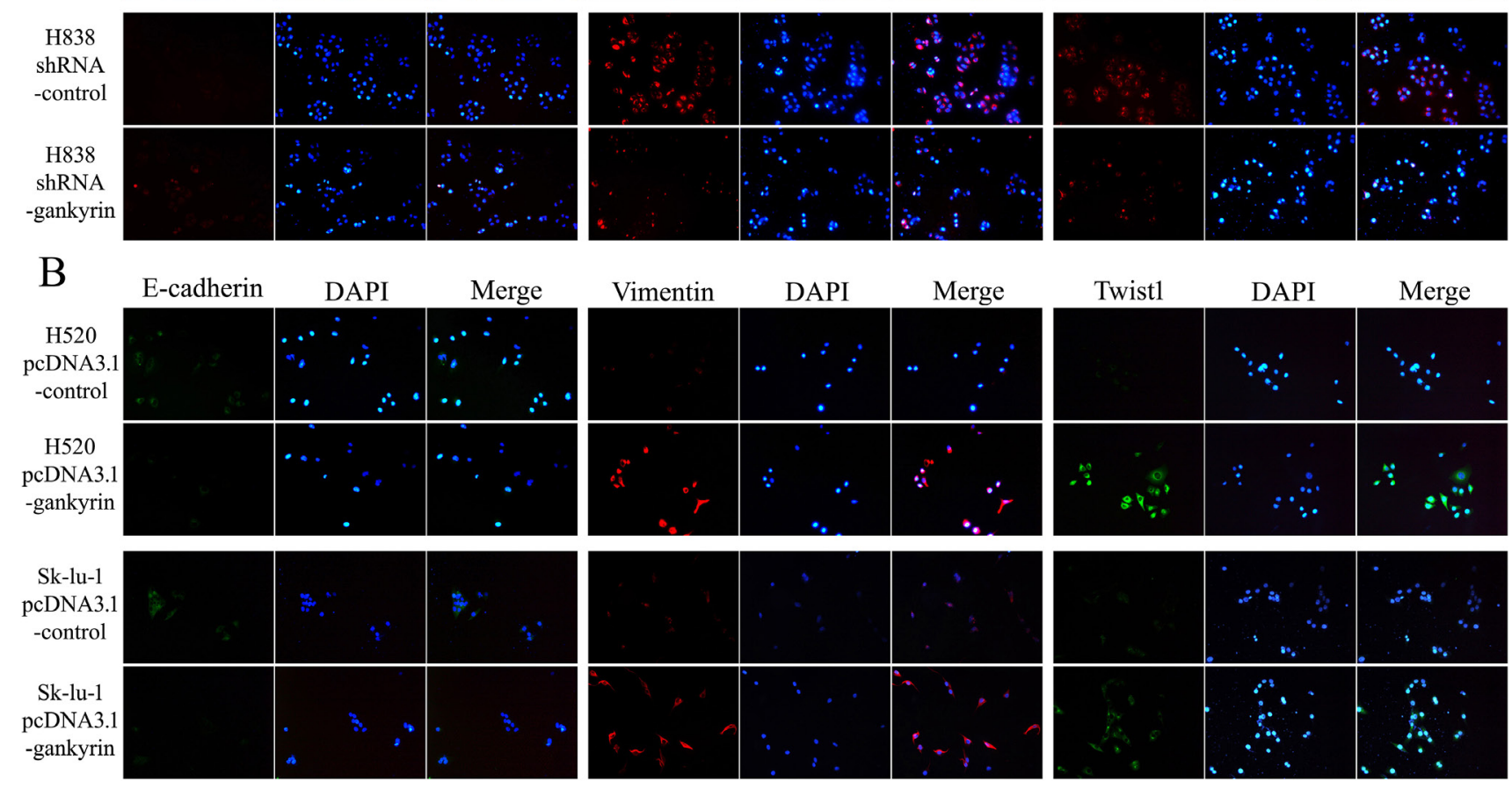

$\mathrm{C}$

H520

H520

pcDNA3.1-gankyrin

$\mathrm{D}$

sk-lu-1

sk-lu-1
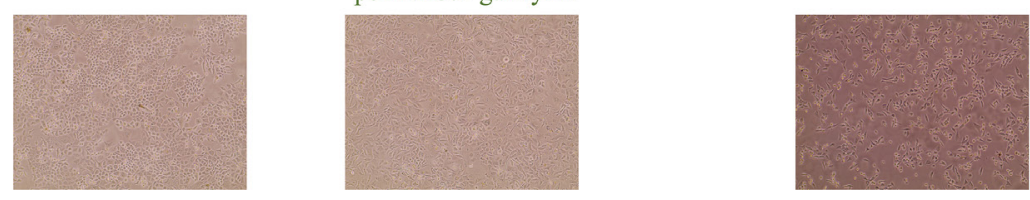

pcDNA3.1-gankyrin

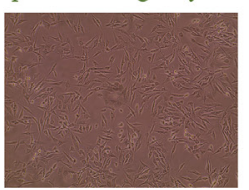

Figure 5: E-cadherin, Vimentin and Twist1 expression were detected both in Gankyrin decreased and increased NSCLC cell lines. (A) The expression of E-cadherin, Vimentin and Twist1 in Gankyrin decreased NSCLC Calu-1 and H838 cell lines. (B) The expression of E-cadherin, Vimentin and Twist1 in Gankyrin increased NSCLC H520 and SK-LU-1 cell lines. (C) The changes of H520 cell shape when Gankyrin protein was stably overexpressed. (D) The changes of sk-lu-1 cell shape when Gankyrin protein was stably overexpressed. 
result, we silenced Gankyrin expression in Sk-lu-1 pCDNA3.1-Gankyrin cells and found that silencing Gankyrin could inhibit phosphorylation of STAT3 as well as EMT induced by TGF- $\beta 1$ (Figure 7D).

To our knowledge, IL-6 is a direct upstream mediator of p-STAT3 in many tumors. Here, we try to investigate whether p-STAT3 was activated by Gankyrin via IL-6 production. IL-6 protein in the conditioned medium of the cultured Sk-lu-1 cells was examined by enzyme-linked immune sorbent assay (ELISA). The results showed that Gankyrin and IL-6 protein level was significantly increased in Gankyrin over-expressed Sk-lu-1 cell line when compared with the control cell line (Figure 7E). These results directly proved that Gankyrin could increase IL-6 expression, and p-STAT3 might be activated by Gankyrin via IL-6 production.

To investigate which molecules played key roles in the Gankyrin-induced EMT process, kinds of downstream molecules such as AKT/p-AKT, STAT3/p-STAT3, SMAD3/p-SMAD3 were evaluated by Western blot assay. The expression levels of p-AKT, p-STAT3 and p-SMAD3 were significantly increased in Gankyrin over-expressed Sk-lu-1 cells, while AKT, STAT3 and SMAD3 levels were not changed at all (Figure 7F). To further explore whether p-AKT was associated with the Gankyrin induced EMT process, inhibitor were used. When p-AKT was inhibited, we found that there was not any change on EMTrelated biomarkers, p-STAT3 and p-SMAD3 expressions (Figure 7G).These data suggested that AKT activation was not correlated with Gankyrin-induced EMT.

To further explore whether Gankyrin could induce EMT through p-STAT3/ p-SMAD3 or p-SMAD3/p-STAT3 in NSCLC, we applied siRNA transduction to knockdown the STAT3 and SMAD3 expression in Sk-lu-1 cell lines, respectively, then detected the EMT-related biomarkers and p-AKT, p-STAT3 and p-SMAD3 expressions by Western blot assay. Interestingly, when p-STAT3 or p-SMAD3 was decreased, EMT-related biomarkers E-cadherin was significantly increased, whereas Vimentin and Twist 1 were significantly decreased, accompanied with down-regulated levels of p-AKT, p-SMAD3 and p-STAT3 (Figure 7H-7I). These data suggested that Gankyrin could activate p-STAT3 or p-SMAD3, forming a closed circle, to induce the EMT. To further verify the role of IL-6 on EMT process, we added exogenous IL-6 ( $2 \mathrm{ng} / \mathrm{L}$ ) into the medium of the cultured Sk-lu-1 cells, The
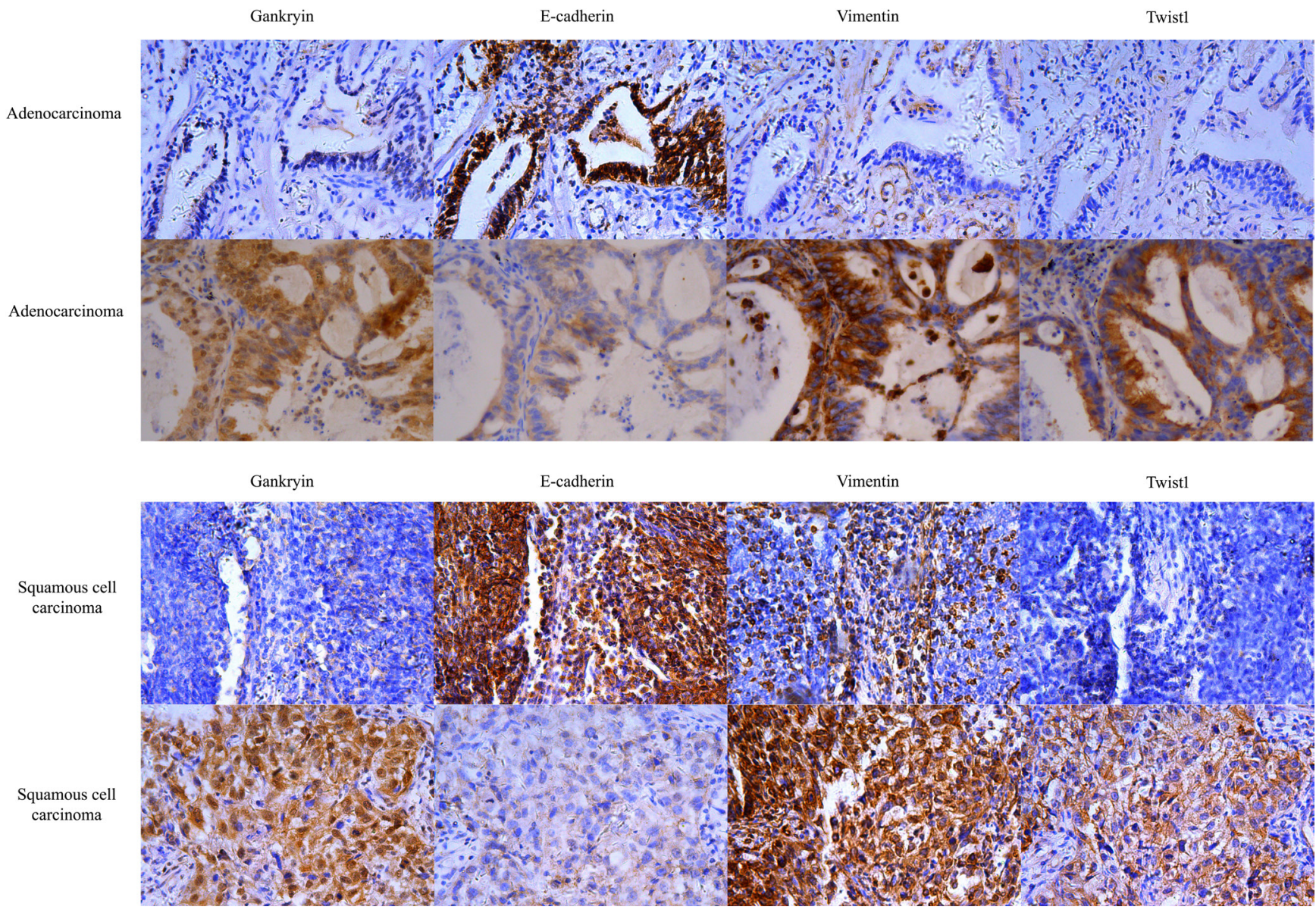

Figure 6: Gankyrin expression correlates with EMT-related biomarkers in NSCLC tissues. (A) EMT-related biomarkers including E-cadherin, Vimentin and Twist1 were detected in ADC tissues using IHC. (B) EMT-related biomarkers including E-cadherin, Vimentin and Twist1 were detected in SCC tissues using IHC. ADC: Adenocarcinoma; SCC: Squamous cell carcinoma. 
results showed that IL-6 could significantly increased the level of Vimentin, Twist1, p-STAT3 and p-SMAD3, and decreased the level of E-cadherin (Figure 7J).

Finally, the percentage of positive expression of p-SMAD3 and p-STAT3 of Gankyrin-positive tumors and Gankyrin-negative tumors in 98 NSCLC tissues were shown in Table 3. Correlation analysis showed that Gankyrin expression in NSCLC tissues had positive correlations with the expression of p-STAT3 $(\mathrm{r}=0.322$, $P=0.002)$ and also had positive correlations with the expression of p-SMAD3 $(r=0.346, P=0.001)$. IHC assay was used to analyze the correlations among Gankyrin, p-SMAD3 and p-STAT3. Representative microphotographs of the expression of Gankyrin, p-SMAD3, p-STAT3 from the same ADC patient and the same SCC patient are shown in Figure 8A-8B. Taken together, these data strongly supported that Gankyrinpromoted EMT and metastasis was partially due to IL-6/pSTAT3 and TGF- $\beta 1 /$ p-SMAD3 pathway activation, which forming a closed circle through Gankyrin.

\section{DISCUSSION}

It has been established that Gankyrin plays critical role in the development and progression of many types of human cancer [28-32, 22]. We also showed that Gankyrin was aberrantly expressed in NSCLC. In our previous study, we revealed that compare to the corresponding normal tissues, Gankyrin is significantly higher expressed in NSCLC tumor tissues. And we found that Gankyrin over-expression was significantly associated with lymphatic metastasis, TNM stage and poor prognosis, and also was an independent prognostic factor of NSCLC [20]. All those results strongly suggested that Gankyrin might represent a biologically cancer exhibiting highlymalignant clinical behavior.

It is well known that the influence of oncoprotein on the prognosis of patients with tumor mainly dependent on two aspects. The first, oncoprotein can promote tumor growth by various molecular pathways to influence the patients' survival; Second, oncoprotein can promote tumor metastasis to influence patients' prognosis. Gankyrin, as an important oncoprotein, has been shown to affect the prognosis of patients with lung cancer, but it is unknown that how Gankyrin will influence the prognosis of patients with lung cancer. Previous study reported that, in lung cancer, increased Gankyrin expression was required for the constitutive activation of Akt and tumorigenesis in those who have ras mutation [33], which indicated that Gankyrin might affect the prognosis of NSCLC partially by promoting tumor growth through activating Akt signal. On the other hand, in our study, the correlation analysis also referred that the Gankyrin over-expression was significantly associated with lymphatic metastasis of NSCLC [20]. So we hypothesized that Gankyrin might also influence the patients' prognosis by promoting
NSCLC metastasis. Our data showed that Gankyrin indeed accelerated the migratory and invasive ability of NSCLC in vitro, and these results supported our hypothesis.

Next in order to further explore potential mechanisms of Gankyrin promoting NSCLC metastasis, we analyzed the relationship between Gankyrin and the EMT-related biomarkers. As far as we known, more and more studies found that EMT could promote carcinoma invasion and metastasis [24]. And recently several studies have shown that EMT was associated with the acquisition of the malignant characteristics of NSCLC cells, and also was an initiation step in tumor metastasis [34-38]. On the other hand, our previous study found that the function of Gankyrin on prognosis was apparent in early stages NSCLC (p-TNM I/II), while not obvious in advanced stage (p-TNM III/IV) patients. So we guessed that Gankyrin might accelerate NSCLC metastasis through participating in the EMT process. The results showed that Gankyrin could induce EMT in NSCLC cell lines, and Gankyrin expression was negatively correlated with E-cadherin, while positively correlated with Vimentin and Twist1 expression in NSCLC tissues. These data strongly supported that Gankyrin was associated with EMT in NSCLC.

The mechanism of EMT is a very complex network and remains to be elusive [39]. However, TGF$\beta 1 / \mathrm{p}-\mathrm{SMAD} 3$ pathway is considered to be a canonical pathway inducing EMT in many tumors, including lung cancer. And some EMT-related oncoprotein or antioncoprotein were proved to mediate EMT by accelerating or attenuating the TGF- $\beta$ signaling pathway [40]. In the present study, we found that Gankyrin-induced EMT was also linked with TGF- $\beta 1$ / p-SMAD3 signal. Our data also suggested that Gankyrin was a downstream molecule of TGF- $\beta 1 /$ p-SMAD3 signaling pathway. In selected lung cancer cell lines, persistently activated or tyrosinephosphorylated STAT3 is partially due to IL-6 induction, and IL-6/p-STAT3 pathway activation is confirmed to be critical for lung cancer progression [41]. In our study, we found that Gankyrin could up-regulate the IL-6 level, and then promote EMT by activating p-STAT3. Some previous study indicated that IL-6/STAT3 signal was required for TGF- $\beta$-induced EMT process in lung cancer cells [42], and the crosstalk between the IL-6/STAT3 and TGF- $\beta /$ p-SMAD3 signaling pathway did exist in certain conditions $[43,44]$. However, we are not clear if there is any interaction. In our study, the results indicated that the crosstalk was partially dependent on Gankyrin, and Gankyrin was a key molecule to sustain EMT phenotype till the cells localized in a new metastatic site even without the continuous IL- 6 and TGF- $\beta$ stimulation. Taken together, our findings were summarized in a model (Figure 7K) to propose that Gankyrin promotes EMT and metastasis in NSCLC through forming a closed circle with IL-6/p-STAT3 and TGF- $\beta /$ p-SMAD3 signal pathway.

In addition, there were some shortcomings presented in this study. First, the sample size is limited. 
Second, we did not investigate the role of Gankyrin on metastasis in vivo, which are very important for oncoprotein validation. Third, there are some other EMT related biomarkers; we just chose the E-cadherin, Vimentin and Twist1 for testing. It will be more convincing if other indicators shall be detected in our experiment. To compensate for these shortcomings, we intend to carry out further multi-center clinical studies, expand the sample size and enrich the means of detection, and add the animal study to strength the conclusion that Gankyrin could promote EMT and metastasis in NSCLC.

\section{MATERIALS AND METHODS}

\section{Patients and tissue samples}

Tissue specimens and detailed information were the same as our previous research [20]. Histological classification of tumors was reviewed by pathologists and based on the World Health Organization criteria. All tumors were staged according to the pathological tumor/ node/metastasis ( $\mathrm{p}$-TNM) classification (7th edition) of the International Union against Cancer [23]. The study protocol was approved by the Regional Ethics Committee for Clinical Research of the Fourth Military Medical University. All patients provided written informed consent for use of their medical records and tissue specimens for research purposes.

\section{Cell lines and treatments}

Non-small cell lung adenocarcinoma cancer cell lines (A549, Sk-lu-1, SPC-A-1, H838), non-small cell lung squamous carcinoma cell lines (H520, Calu-1) and human bronchial epithelial cells (HBE) were preserved in our laboratory. A549, Sk-lu-1, SPC-A-1, H838, H520 and Calu- 1 cell lines were maintained at $37^{\circ} \mathrm{C}$ in a humidified incubator containing 5\% $\mathrm{CO}_{2}$, in RPMI 1640 with supplemented with $10 \%$ fetal bovine serum and $1 \%$ penicillin/streptomycin. HBE cell line were maintained at $37^{\circ} \mathrm{C}$ in a humidified incubator containing $5 \% \mathrm{CO}_{2}$, in high sugar Dulbecco's Modified Eagle Medium supplemented with $10 \%$ fetal bovine serum and $1 \%$ penicillin/streptomycin. TGF- $\beta 1$ (ProSpec, 10 ng/ml)/IL-6 (PeproTech, 2 ng/L) was dissolved in DMSO (Sigma, St. Louis, MO) and stored in small aliquots at $-20^{\circ} \mathrm{C}$.
A

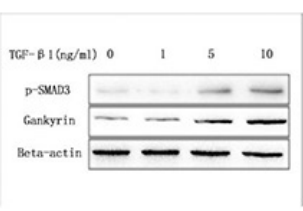

E

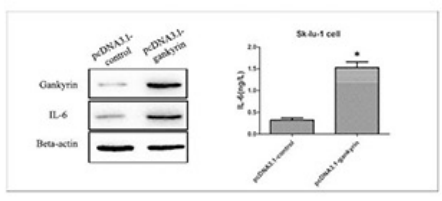

I

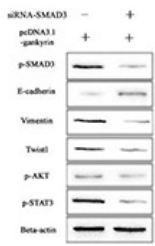

B

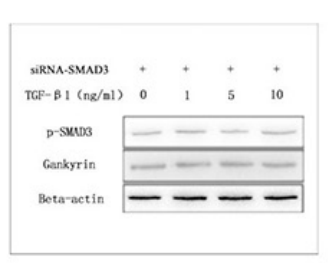

F

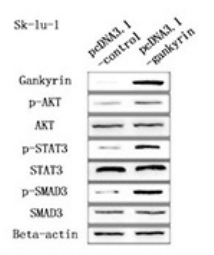

$\mathrm{J}$

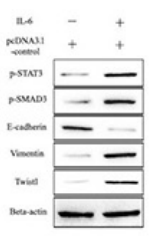

C

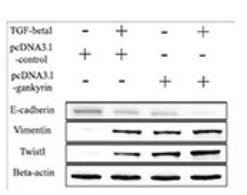

G

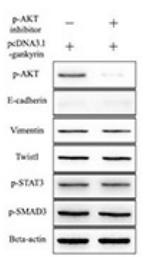

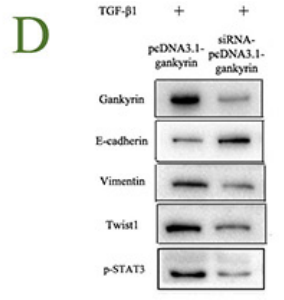
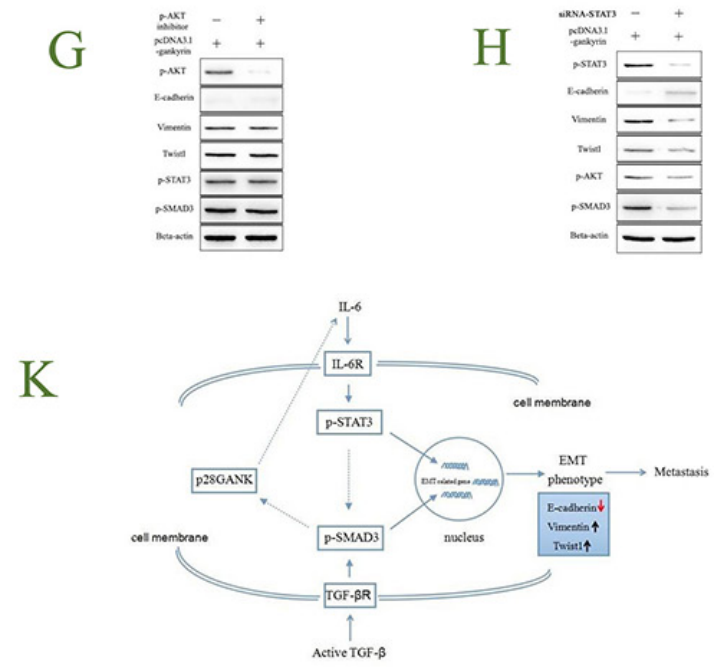

Figure 7: Gankyrin-promoted EMT is partially due to IL-6/p-STAT3 and TGF- $\beta 1 / p-S M A D 3$ pathway activation. (A) TGF- $\beta 1$ could regulate p-SMAD3 and Gankyrin expressions. (B) TGF- $\beta 1$ could regulate Gankyrin expression through p-SMAD3. (C) Gankyrin promotes the process of TGF- $\beta 1$ induced EMT. (D) Silencing Gankyrin inhibits EMT and p-STAT3 induce by TGF- $\beta 1$. (E) Gankyrin over-expression could up-regulate IL-6 level. (F) Gankyrin could increase p-AKT, p-STAT3, p-SMAD3 expressions. (G) The process of TGF- $\beta 1$ induced EMT has nothing to do with p-AKT. (H-J) STAT3 and SMAD3 could promote the process of Gankyrin induced EMT. (K) IL-6 induces EMT through activating STAT3/SMAD3 in NSCLC. 


\section{Immunohistochemistry}

The tumor samples were fixed with $10 \%$ formaldehyde and embedded with paraffin. Sections were sliced up at $4 \mu \mathrm{m}$ thickness, deparaffinized with a series of xylene and rehydrated through a graded series of alcohol. Microwave antigen retrieval was performed at $750 \mathrm{~W}$ for $5 \mathrm{~min}$ and $450 \mathrm{~W}$ for $15 \mathrm{~min}$ in citrate buffer (pH 6.0) to enhance the immuno-reactivity. After blocking the endogenous peroxidase activity with $3 \%$ hydrogen peroxidase for $30 \mathrm{~min}$, sections were incubated with 5\% normal goat serum for $30 \mathrm{~min}$ at room temperature to block nonspecific antibody reaction. After washing the tissue samples with PBS three times for $5 \mathrm{~min}$, sections were incubated with the primary antibodies (Gankyrin, 1:50, Santa Cruz Biotechnology, Inc. USA; p-SMAD3, 1:100, Abcam, Cambridge, UK; p-STAT3, 1:100, Cell Signaling, Inc. USA) overnight at $4^{\circ} \mathrm{C}$, and incubated with an EnVision ${ }^{\mathrm{TM}}$ Detection Kit (DAKO, Denmark) following the manufactures' instruction. The sections were then reacted with $0.003 \% 3$, 30-diaminobenzidine and counterstained with hematoxylin. To confirm the specificity of the immunostaining, negative controls were obtained by replacing the primary antibody with PBS.

\section{Evaluation of immunohistochemical staining}

Five random fields from each section were viewed under a light microscope (Leica DM4000B, Germany) at $\times 200$ magnification. The expression of protein was scored by multiplication of the percentage of positive tumor cells and the staining intensity $[20,24,25]$.

\section{Plasmid construction and transfection}

For Gankyrin overexpression, the whole cDNA sequence of Gankyrin was cloned into the Hind III and $\mathrm{Bam} \mathrm{HI}$ site of $\mathrm{pCDNA}(+) 3.1$ vector (Invitrogen, Carlsbad, CA) and transformed into DH5 $\alpha$ competent cells. Positive clones were identified and verified by using restrictive cleavage and sequenced.

H520 and SK-LU-1 cell lines were plated into sixwell plates at $5 \times 10^{5}$ cells per well. After $24 \mathrm{~h}$, cells were transfected with $2 \mu \mathrm{g}$ or $4 \mu \mathrm{g}$ Gankyrin expression vector using Lipofectamine 2000 (Invitrogen, Carlsbad, CA) according to the manufacturer's instruction. Stable clones were isolated after selection with $800 \mu \mathrm{g} / \mathrm{ml}$ of G418 for 1 week, and then $400 \mu \mathrm{g} / \mathrm{ml}$ of G418 for 3 weeks.

\section{Lentivirus vectors for Gankyrin RNA interference}

To observe the effect of inhibiting Gankyrin on human NSCLC cells, we employed the newly developed lentivirus-delivered RNA interfering technique. hU6MCS-CMV-EGFP-Lentivirus (GeneChem, China) was used to express small interfering RNAs (siRNAs) targeting the Gankyrin sequence (Genbank No.5716). A non-targeting sequence was used as a lentivirus negative control (NC). Targeted oligonucleotide sequences were: siRNA-1: GGTTGGTCTCCTCTTCATA; siRNA-2: CAGCTTGGATTTATTCTTA; siRNA-3: GTTACTTGT TCGAAGCTTA and TTCTCCGAACGTGTCACGT for the lentivirus negative control. Preliminary experiment results showed that siRNA-1 significantly could inhibited

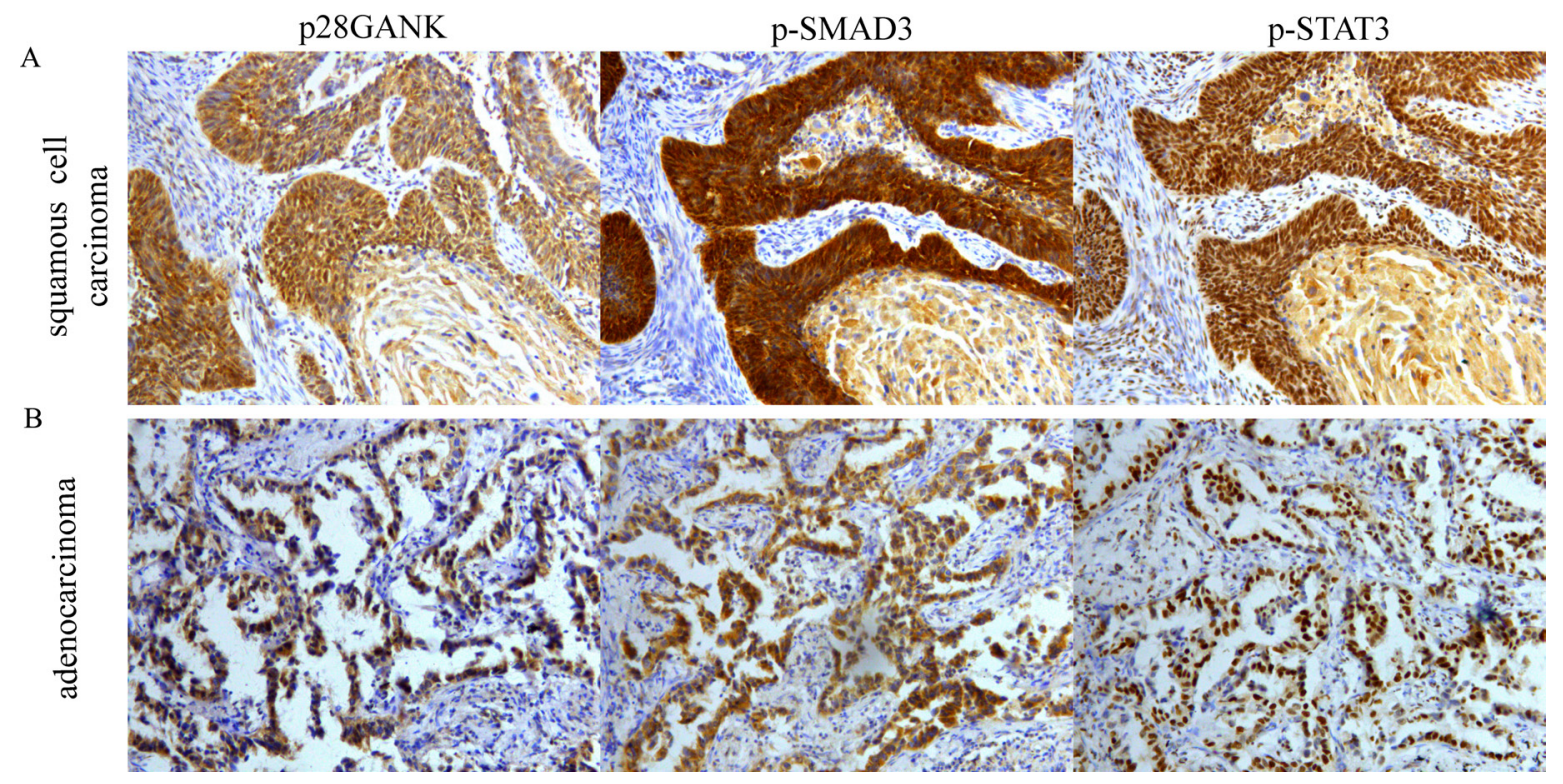

Figure 8: Correlation of Gankyrin expression with p-SMAD3 and p-STAT3 in NSCLC tissues using IHC. (A) Correlation of Gankyrin expression with p-SMAD3 and p-STAT3 from the same SCC tissues. (B) Correlation of Gankyrin expression with p-SMAD3 and p-STAT3 from the same ADC tissues. 
Gankyrin expression, therefore, siRNA-1 was selected as an optimal siRNA for following experiments. NSCLC cell lines, Calu-1 (SCC) and H838 (ADC), were infected with Gankyrin-siRNA lentivirus and with NC lentivirus. Cells were plated in 6 -well plates $\left(5 \times 10^{4}\right.$ cells/well $)$, grown to $60 \%$ confluence, and treated with tittered viral supernatant ata multiplicity of infection (MOI) of 20 for $12 \mathrm{~h}$ without toxic effect observed. Then, the media was changed to RPMI 1640 medium supplemented with 10\% FBS. The interference efficiency of the template was detected by RTPCR and Western blot analysis. The Calu-1 and H838 cells were transfected with the Gankyrin-siRNA lentivirus or $\mathrm{NC}$ lentivirus were designated as Calu-1-siRNA or Calu1-NC and H838-siRNA or H838-NC, respectively. Non transfected cells were also included as a positive control and designated Calu-1-Control or H838-Control.

\section{Purification of total cellular mRNA and semi- quantitative RT-PCR}

Total RNA was extracted from the fresh tissue specimen of NSCLC patients using E.Z.N.A. ${ }^{\circledR}$ Total RNA Kit I (Omega Bio-TekInc., Georgia, USA) according to the manufacturer's protocols. Reverse transcription of total cellular RNA was performed using a RevertAid FirstStrand cDNA Synthesis Kit (Thermo scientific, Vilnius, Lithuania). cDNA was subjected to PCR for 35 cycles of amplification using an cDNA PCR kit (CWbiotech, Peking, China). Each PCR cycle consisted of a denaturation step for $45 \mathrm{~s}$ at $95^{\circ} \mathrm{C}$, an annealing step for $35 \mathrm{~s}$ at $62^{\circ} \mathrm{C}$, and an extension step for $50 \mathrm{~s}$ at $72^{\circ} \mathrm{C}$. The PCR products were separated on $1.0 \%$ agarose gel and visualized by ethidium bromide staining. $\beta$-actin mRNA was used as an internal control for semi-quantitative analyses of Gankyrin mRNA. The PCR primers used for Gankyrin were 5'-AGCAGCCAAGGGTAACTTGA-3' as the forward primer and 5'-TACTTGCTCCTTGGGACACC-3' as the reverse primer; and for $\beta$-actin, 5'-CTCCATCCTGGCCT CGCTGT-3' was used as the forward primer and 5'-GCTGTCACCTTCACCGTTCC-3' as the corresponding reverse primer.

\section{Protein isolation and western blot}

Cytoplasmic and nuclear protein were extracted from cells using RIPA lysate (P0013B, Beyotime) and Western Blot were performed using anti-Gankyrin specific monoclonal antibody (Santa Cruz Biotechnology, Inc. USA), p-SMAD3 monoclonal antibody (Ser423/425, Abcam), p-STAT3 monoclonal antibody (D3A7, Cell Signaling) and the $\beta$-actin specific polyclonal antibody (CW0097, CWbiotech).

Protein concentrations were determined using the BCA assay kit (Pierce). Equivalent amounts of each protein sample were mixed with loading buffer (CW0027A, CWBIO), heated at $65^{\circ} \mathrm{C}$ for $30 \mathrm{~min}$, and subjected to SDS-PAGE using 12\% separation gel and $5 \%$ spacer gel. Then protein was transferred to a PVDF membrane (Solarbio) by electro blotting (Bio-RAD). The membrane was blocked for 3 hours at room temperature in TBST (25 mM Tris/ $\mathrm{HCl}, \mathrm{pH} 7.5,150 \mathrm{mM} \mathrm{NaCl}$, $0.1 \%$ Tween 20 ) containing $5 \%$ nonfat dry milk. The membranes were incubated overnight at $4^{\circ} \mathrm{C}$ in $1: 200$ dilution of anti-Gankyrin monoclonal antibody, 1:1000 dilution of SMAD3 monoclonal antibody, 1:2000 dilution of p-SMAD3 monoclonal antibody, 1:1000 dilution of STAT3 monoclonal antibody, 1:2000 dilution of p-STAT3 monoclonal antibody, 1:500 dilution of AKT monoclonal antibody, 1:1000 dilution of p-AKT monoclonal antibody, 1:500 dilution of E-cadherin monoclonal antibody, 1:2000 dilution of Vimentin monoclonal antibody, 1:300 dilution of Twist1 monoclonal antibody, 1:500 dilution of IL-6 monoclonal antibody and 1:2500 dilution of $\beta$-actin polyclonal antibody with WB Antibody Diluent (P0023A, Beyotime), washed six times in TBST, and incubated for $35 \mathrm{~min}$ in a 1:5000 dilution of goat anti-rabbit Ig-HRP (EK020, Zhuangzhibio) with WB Secondary Antibody Diluent (P0023D, Beyotime). Immunoreactive bands were revealed by the enhanced chemiluminescence system (Santa Cruz Biotechnology, Santa Cruz, CA). Pictures were photographed and analyzed by Gel Dox XR system (Bio-Rad, Philadelphia, PA).

\section{Scratch wound healing assay}

Cell migration was measured using a scratch assay determined, as described previously [26]. After 5 days of lentiviral infection, both transfected and untreated Calu-1 and H838 cells were harvested and their $5 \times 10^{5}$ cells were plated in 6-well plates. Cells were incubated overnight. After incubation, the plates yielded cells of $80 \%$ confluence, the monolayer was scraped in a straight line to create a "scratch" using a $200 \mu \mathrm{L}$ pipette tip. After removing debris and adding fresh media containing no FBS, cells were photographed at $0 \mathrm{~h}, 24 \mathrm{~h}$, and $48 \mathrm{~h}$. The migration distance was measured and assessed using image $\mathbf{J}$ software at 3 different sites from each wound area of scratch, at each time point. The healing degree was calculated by cell relative migration area for each treatment.

\section{Transwell invasion and migration assay}

To further examined the cell invasion and migration, the transwell invasion and migration was performed using $8 \mu \mathrm{m}$ pore size transwell chambers (Corning, USA) in vitro following the manufacturer's instructions. In brief, the matrigel (5 mg/ml, Corning, USA) was diluted into $1 \mathrm{mg} / \mathrm{ml}$ in ice-cold RPMI 1640 medium supplemented with $10 \%$ FBS. An aliquot of $200 \mu \mathrm{L}$ diluted matrigel was added to the upper transwell chambers and incubated at $37^{\circ} \mathrm{C}$ for $4 \mathrm{~h}$ for gelling. A total of $1 \times 10^{5}$ cells in $400 \mu \mathrm{L}$ 
media supplemented with no FBS were plated in the upper chamber and $600 \mu \mathrm{L}$ RPMI 1640 medium supplemented with $10 \%$ FBS was covered on the bottom chambers as chemo attractant. After incubation at $37^{\circ} \mathrm{C}$ for $48 \mathrm{~h}$, the non-invasive cells in the top surface were carefully removed with a cotton swab. The invasive cells that had traversed to the bottom surface were fixed in dehydrated alcohol for $30 \mathrm{~min}$ and stained with $4 \mathrm{mg} / \mathrm{ml}$ crystal violet for $10 \mathrm{~min}$. To quantify the traversed cells, cell counting was obtained by photographing 5 random fields under microscope at $400 \times$ magnification [27]. The migration assay was performed in a similar strategy with chamber membrane without coating with matrigel.

\section{ELISA}

Supernatants from NSCLC cells were collected and IGF1 ELISA was subsequently performed using IGF1 ELISA Kit and following the manufacturer's instructions.

\section{Immunofluorescence (IF)}

Cells were fixed in 4\% paraformaldehyde for $30 \mathrm{~min}$ and blocked in $0.5 \%$ Trion X-100 for $15 \mathrm{~min}$. The cells were washed 3 times ( 5 min for each) with PBS after each step. The tumor samples were fixed with $10 \%$ formaldehyde and embedded inparaffin. Sections were sliced at $4 \mu \mathrm{m}$ thickness, deparaffinized with a series of xylene washes, and rehydrated through a graded series of alcohol rinses. Microwave antigen retrieval was performed at $750 \mathrm{~W}$ for $5 \mathrm{~min}$ and $450 \mathrm{~W}$ for $15 \mathrm{~min}$ in $0.1 \mathrm{M}$ citrate buffer ( $\mathrm{pH}$ 6.0) to enhance the immunoreactivity. To block the endogenous peroxidase activity, all the cells and tumor samples were incubated in $3 \%$ hydrogen peroxidase at room temperature for $30 \mathrm{~min}$ and washed with PBS three times for $5 \mathrm{~min}$. All the cells and tumor samples were incubated with $10 \%$ normal goat serum for $30 \mathrm{~min}$ at room temperature to block nonspecific antibody reaction, followed by incubation in a humidified chamber overnight at $4^{\circ} \mathrm{C}$ with anti-Gankyrin rabbit polyclonal antibody (1:50, Santa Cruz Biotechnology). After an additional series of washes, the samples were stained with goat anti-rabbit (Cy3, Zhuangzhi bio) or were stained with goat anti-rabbit (Alexa Fluor 488, Zhuangzhi bio) at $37^{\circ} \mathrm{C}$ for $4 \mathrm{~min}$, then each sample was washed with PBS three times, for $5 \mathrm{~min}$. Then, the samples were incubated with 4'6-diamino-2-phenylindole (DAPI) antibody (1:160, Abcam) for 5-10 min at room temperature. After the final washing, the samples were mounted in 50\% glycerol (in PBS) and visualized under a fluorescence microscope (Leica DM4000B, Germany).

\section{Statistical analysis}

Each experiment was performed in triplicate. Bands from Western blot were quantized using Quantity One software (Bio-Rad, USA). Relative protein levels were calculated relative to the amount of $\beta$-actin respectively. All statistical analyses were performed with SPSS 18.0 software (SPSS, Inc., Chicago, IL). All values in the text and figures are expressed as the mean \pm SD of these observations. Student's $t$-test was used for raw data analysis. A $P$-value $<0.05$ was considered statistically significant.

\section{ACKNOWLEDGMENTS}

The work was supported by the grant from the National Natural Science Foundation of China (30973379 to Xiao-fei Li). We also thank Hao Xing, Miao-miao Wen, Jing-hua Xia, Liang-bo Fan and Guang Yang (Thoracic surgery department laboratory) for their help in providing experimental technical support. Finally, we would like to thank all members of our research team, who collaborated to ensure the study proceeded smoothly.

\section{CONFLICTS OF INTEREST}

The authors declare no conflicts of interest.

\section{FUNDING}

This work had no specific funding.

\section{REFERENCES}

1. She J, Yang P, Hong Q, Bai C. Lung cancer in China: challenges and interventions. Chest. 2013; 143:1117-1126.

2. Chen DM, Mao KY, Yang L, Jiang HB. Status of anti-lung cancer drug patents applications in China from 2003 to 2012. Recent patents on anti-cancer drug discovery. 2014; 9:221-240.

3. Parkin DM, Bray F, Ferlay J, Pisani P. Global cancer statistics, 2002. CA Cancer J Clin. 2005; 55:74-108.

4. Devesa SS, Bray F, Vizcaino AP, Parkin DM. International lung cancer trends by histologic type: male:female differences diminishing and adenocarcinoma rates rising. International journal of cancer. 2005; 117:294-299.

5. Herbst RS, Heymach JV, Lippman SM. Lung cancer. The New England journal of medicine. 2008; 359:1367-1380.

6. Thiery JP, Acloque H, Huang RY, Nieto MA. Epithelialmesenchymal transitions in development and disease. Cell. 2009; 139:871-890.

7. Hou M, Cheng Z, Shen H, He S, Li Y, Pan Y, Feng C, Chen X, Zhang Y, Lin M, Wang L, Ke Z. High expression of CTHRC1 promotes EMT of epithelial ovarian cancer (EOC) and is associated with poor prognosis. Oncotarget. 2015; 6:35813-35829. doi: 10.18632/oncotarget.5358.

8. Denlinger CE, Ikonomidis JS, Reed CE, Spinale FG. Epithelial to mesenchymal transition: the doorway to metastasis in human lung cancers. J Thorac Cardiovasc Surg. 2010; 140:505-13. 
9. Nurwidya F, Takahashi F, Murakami A, Takahashi K. Epithelial mesenchymal transition in drug resistance and metastasis of lung cancer. Cancer Res Treat. 2012; 44:151-6.

10. Soltermann A. Epithelial-mesenchymal transition in nonsmall cell lung cancer. Pathologe. 2012; 33:311-7.

11. Lindsey S, Langhans SA. Crosstalk of Oncogenic Signaling Pathways during Epithelial-Mesenchymal Transition. Frontiers in oncology. 2014; 4: 358.

12. De Craene B, Berx G. Regulatory networks defining EMT during cancer initiation and progression. Nature reviews Cancer. 2013; 13:97-110.

13. Higashitsuji H, Itoh K, Nagao T, Dawson S, Nonoguchi K, Kido T, Mayer RJ, Arii S, Fujita J. Reduced stability of retinoblastoma protein by gankyrin, an oncogenic ankyrin-repeat protein overexpressed in hepatomas. Nature medicine. 2000; 6:96-99.

14. Yang C, Tan YX, Yang GZ, Zhang J, Pan YF, Liu C, Fu J, Chen Y, Ding ZW, Dong LW, Wang HY. Gankyrin has an antioxidative role through the feedback regulation of Nrf2 in hepatocellular carcinoma. The Journal of experimental medicine. 2016; 213:859-875.

15. Zhao X, Fu J, Xu A, Yu L, Zhu J, Dai R, Su B, Luo T, Li N, Qin W, Wang B, Jiang J, Li S, et al. Gankyrin drives malignant transformation of chronic liver damage-mediated fibrosis via the Rac1/JNK pathway. Cell death \& disease. 2015; 6: e1751.

16. Zhen C, Chen L, Zhao Q, Liang B, Gu YX, Bai ZF, Wang K, Xu X, Han QY, Fang DF, Wang SX, Zhou T, Xia Q, et al. Gankyrin promotes breast cancer cell metastasis by regulating Rac1 activity. Oncogene. 2013; 32:3452-3460.

17. Qin X, Wang X, Liu F, Morris LE, Wang X, Jiang B, Zhang Y. Gankyrin activates mTORC1 signaling by accelerating TSC2 degradation in colorectal cancer. Cancer letters. 2016; 376:83-94.

18. Pei T, Li Y, Wang J, Wang H, Liang Y, Shi H, Sun B, Yin D, Sun J, Song R, Pan S, Sun Y1, Jiang H, et al. YAP is a critical oncogene in human cholangiocarcinoma. Oncotarget. 2015; 6:17206-17220. doi: 10.18632/ oncotarget. 4043 .

19. Chen J, Bai M, Ning C, Xie B, Zhang J, Liao H, Xiong J, Tao X, Yan D, Xi X, Chen X, Yu Y, Bast RC, et al. Gankyrin facilitates follicle-stimulating hormone-driven ovarian cancer cell proliferation through the PI3K/AKT/HIF1alpha/cyclin D1 pathway. Oncogene. 2015; 35:2506-2517.

20. Wang WP, Yan XL, Li WM, Ni YF, Zhao JB, Lu Q, Wang XJ, Sun Y, Chen P, Yan BY, Cui Y, Zhang ZP, Li XF. Clinicopathologic features and prognostic implications of Gankyrin protein expression in non-small cell lung cancer. Pathology, research and practice. 2015; 211:939-947.

21. Chattopadhyay A, O'Connor CJ, Zhang F, Galvagnion C, Galloway WR, Tan YS, Stokes JE, Rahman T, Verma C, Spring DR, Itzhaki LS. Discovery of a small-molecule binder of the oncoprotein gankyrin that modulates gankyrin activity in the cell. Scientific reports. 2016; 6:23732.
22. Zheng T, Hong X, Wang J, Pei T, Liang Y, Yin D, Song R, Song X, Lu Z, Qi S, Liu J, Sun B, Xie C, et al. Gankyrin promotes tumor growth and metastasis through activation of IL-6/STAT3 signaling in human cholangiocarcinoma. Hepatology (Baltimore, Md). 2014; 59:935-946.

23. Goldstraw P, Crowley J, Chansky K, Giroux DJ, Groome PA, Rami-Porta R, Postmus PE, Rusch V, Sobin L. The IASLC Lung Cancer Staging Project: proposals for the revision of the TNM stage groupings in the forthcoming (seventh) edition of the TNM Classification of malignant tumours. Journal of thoracic oncology. 2007; 2:706-714.

24. Gastl G, Spizzo G, Obrist P, Dunser M, Mikuz G. Ep-CAM overexpression in breast cancer as a predictor of survival. Lancet (London, England). 2000; 356:1981-1982.

25. Zhao J, Zhou Y, Zhang Z, Tian F, Ma N, Liu T, Gu Z, Wang Y. Upregulated fascin1 in non-small cell lung cancer promotes the migration and invasiveness, but not proliferation. Cancer letters. 2010; 290:238-247.

26. Liang CC, Park AY, Guan JL. In vitro scratch assay: a convenient and inexpensive method for analysis of cell migration in vitro. Nature protocols. 2007; 2:329-333.

27. Wang L, Yang HJ, Gao SS, Wang M, Shi Y, Cheng BF, Feng ZW. Identification of a novel role of RING finger protein 11 promoting the metastasis of murine melanoma cells. American journal of translational research. 2015; 7:1629-1635.

28. Jing $\mathrm{H}$, Zhang G, Meng L, Meng Q, Mo H, Tai Y. Gradually elevated expression of Gankyrin during human hepatocarcinogenesis and its clinicopathological significance. Scientific reports. 2014; 4:5503.

29. Ortiz CM, Ito T, Tanaka E, Tsunoda S, Nagayama S, Sakai Y, Higashitsuji H, Fujita J, Shimada Y. Gankyrin oncoprotein overexpression as a critical factor for tumor growth in human esophageal squamous cell carcinoma and its clinical significance. International journal of cancer. $2008 ; 122: 325-332$.

30. Bai Z, Tai Y, Li W, Zhen C, Gu W, Jian Z, Wang Q, Lin JE, Zhao Q, Gong W, Liang B, Wang C, Zhou T. Gankyrin activates IL-8 to promote hepatic metastasis of colorectal cancer. Cancer research. 2013; 73:4548-4558.

31. Meng Y, He L, Guo X, Tang S, Zhao X, Du R, Jin J, Bi Q, Li H, Nie Y, Liu J, Fan D. Gankyrin promotes the proliferation of human pancreatic cancer. Cancer letters. 2010; 297:9-17.

32. Kim YH, Kim JH, Choi YW, Lim SK, Yim H, Kang SY, Chung YS, Lee GY, Park TJ. Gankyrin is frequently overexpressed in breast cancer and is associated with ErbB2 expression. Experimental and molecular pathology. 2013; 94:360-365.

33. Man JH, Liang B, Gu YX, Zhou T, Li AL, Li T, Jin BF, Bai B, Zhang HY, Zhang WN, Li WH, Gong WL, Li HY, et al. Gankyrin plays an essential role in Ras-induced tumorigenesis through regulation of the RhoA/ROCK pathway in mammalian cells. The Journal of clinical investigation. 2010; 120:2829-2841. 
34. Feng J, Zhang X, Zhu H, Wang X, Ni S, Huang J. FoxQ1 overexpression influences poor prognosis in non-small cell lung cancer, associates with the phenomenon of EMT. PloS one. 2012; 7: e39937.

35. Tischler V, Pfeifer M, Hausladen S, Schirmer U, Bonde AK, Kristiansen G, Sos ML, Weder W, Moch H, Altevogt P, Soltermann A. L1CAM protein expression is associated with poor prognosis in non-small cell lung cancer. Molecular cancer. 2011; 10:127.

36. Yang J, Weinberg RA. Epithelial-mesenchymal transition: at the crossroads of development and tumor metastasis. Developmental cell. 2008; 14:818-829.

37. Gao L, Xie H, Dong L, Zou J, Fu J, Gao X, Ou L, Xiang S, Song H. Gankyrin is essential for hypoxia enhanced metastatic potential in breast cancer cells. Molecular medicine reports. 2014; 9:1032-1036.

38. Song X, Wang J, Zheng T, Song R, Liang Y, Bhatta N, Yin D, Pan S, Liu J, Jiang H, Liu L. LBH589 Inhibits proliferation and metastasis of hepatocellular carcinoma via inhibition of gankyrin/STAT3/Akt pathway. Molecular cancer. 2013; 12:114.

39. Thiery JP, Sleeman JP. Complex networks orchestrate epithelial-mesenchymal transitions. Nature reviews Molecular cell biology. 2006; 7:131-142.

40. Risolino $\mathrm{M}$, Mandia $\mathrm{N}$, Iavarone $\mathrm{F}$, Dardaei L, Longobardi E, Fernandez S, Talotta F, Bianchi F, Pisati F, Spaggiari L, Harter PN, Mittelbronn M,Schulte D, et al.
Transcription factor PREP1 induces EMT and metastasis by controlling the TGF-beta-SMAD3 pathway in non-small cell lung adenocarcinoma. Proceedings of the National Academy of Sciences of the United States of America. 2014; 111:E3775-3784.

41. Qu Z, Sun F, Zhou J, Li L, Shapiro SD, Xiao G. Interleukin-6 Prevents the Initiation but Enhances the Progression of Lung Cancer. Cancer research. 2015; 75:3209-3215.

42. Liu RY, Zeng Y, Lei Z, Wang L, Yang H, Liu Z, Zhao J, Zhang HT. JAK/STAT3 signaling is required for TGF-betainduced epithelial-mesenchymal transition in lung cancer cells. International journal of oncology. 2014; 44:1643-1651.

43. Zhang XL, Topley N, Ito $\mathrm{T}$, Phillips A. Interleukin-6 regulation of transforming growth factor (TGF)-beta receptor compartmentalization and turnover enhances TGFbeta1 signaling. The Journal of biological chemistry. 2005; 280:12239-12245.

44. Abulaiti A, Shintani Y, Funaki S, Nakagiri T, Inoue M, Sawabata N, Minami M, Okumura M. Interaction between non-small-cell lung cancer cells and fibroblasts via enhancement of TGF-beta signaling by IL-6. Lung cancer (Amsterdam, Netherlands). 2013; 82:204-213. 